\title{
Spin wave excitations: The main source of the temperature dependence of interlayer exchange coupling in nanostructures
}

\author{
S. Schwieger, ${ }^{1,2}$ J. Kienert, ${ }^{2, \text { * }}$ K. Lenz, ${ }^{3}$ J. Lindner, ${ }^{3, \text {, }}$ K. Baberschke, ${ }^{3}$ and W. Nolting ${ }^{2}$ \\ ${ }^{1}$ Technische Universität Ilmenau, Theoretische Physik I, Postfach 1005 65, 98684 Ilmenau, Germany \\ ${ }^{2}$ Lehrstuhl Festkörpertheorie, Institut für Physik, Humboldt-Universität zu Berlin, Newtonstr. 15, 12489 Berlin, Germany \\ ${ }^{3}$ Institut für Experimentalphysik, Freie Universität Berlin, Arnimallee 14, 14195 Berlin, Germany
}

\begin{abstract}
Quantum mechanical calculations based on an extended Heisenberg model are compared with ferromagnetic resonance (FMR) experiments on prototype trilayer systems $\mathrm{Ni}_{7} / \mathrm{Cu}_{n} / \mathrm{Co}_{2} / \mathrm{Cu}(001)$ in order to determine and separate for the first time quantitatively the sources of the temperature dependence of interlayer exchange coupling. Magnon excitations are responsible for about $75 \%$ of the reduction of the coupling strength from zero to room temperature. The remaining $25 \%$ are due to temperature effects in the effective quantum well and the spacer/magnet interfaces.
\end{abstract}

PACS numbers: 75.10.-b,75.70.Cn,76.50.+g,75.30.Ds

The coupling of two magnetic layers through a nonmagnetic spacer layer (interlayer exchange coupling, IEC) has received considerable attention recently. The physics at $T=0$ can be described quite generally in the framework of a quantum interference model [1, 2, 3], e.g. the dependence on the spacer thickness. Within this theory, the period, the amplitude, and the phase of the coupling are well understood today. However, most of the experiments on magnetic multilayers and their IEC have been undertaken at ambient temperature, not only for easier feasibility but also in view of technological applications. In this Letter we evidently show in theory and experiment that the major effect is due to magnon excitations, whereas band structure effects are less important.

Much experimental and theoretical work focused on the oscillating dependence of the IEC on the spacer thickness resulting in ferromagnetic (FM) and antiferromagnetic (AFM) coupling of the magnetic layers [4]. As for the temperature dependence there is convincing experimental evidence on several IEC systems that the coupling strength decreases with $T$ from its $T=0$-value to almost zero at $T=T_{\mathrm{C}}$ in metallic systems $5,[6,6]$ and that it shows an effective $T^{3 / 2}$-dependence [5]. However relatively little theoretical work has been carried out concerning the temperature dependence of the IEC over the full temperature range. The detailed ab-initio investigations which focused on Fermi edge softening effects are restricted to low temperatures by the assumption that the spin-resolved band structure does not change significantly [8, 9]. Working at all temperatures, Mills and coworkers investigated the influence of spin waves and predicted the experimentally found $T^{3 / 2}$-dependence [5, 10].

In this Letter we present a novel approach which combines a quantum mechanical treatment of magnetic multilayers at all temperatures with experiments on the prototype IEC systems $\mathrm{Ni}_{7} / \mathrm{Cu}_{n} / \mathrm{Co}_{2} / \mathrm{Cu}(001)$ (subscript: number of layers). Up to now a quantitative separation of the possible sources of the $T$-dependence (spacer ef- fects like softening of the Fermi surface or reduction of the spin asymmetry of the reflection coefficients vs. magnetic layer effect, i.e. excitation of magnons) has not been achieved [11]. The key benefit of the current approach is the possibility to "switch on and off" magnon excitations in our model. Thus, by performing one-parameter fits of the microscopic interlayer exchange coupling with and without magnons, we can separate the two main sources of the temperature dependence of the IEC. We find that the magnetic contribution exceeds the spacer part by a factor of 3 in the range from $\approx 0.15 \cdot T_{\mathrm{C}}$ to $T_{\mathrm{C}}$.

The theoretical description of $T$-dependent IEC experiments often relies on the classical, macroscopic LandauLifshitz (LL) equations. This continuum's approach is usually used together with an expansion of the free energy to obtain the IEC energy by defining [11, 12]

$$
2 J_{\text {inter }}(T)=F_{\mathrm{IEC}}(T)=F_{\uparrow \uparrow}(T)-F_{\uparrow \downarrow}(T) .
$$

Although the LL approach allows for an examination of the overall $T$-dependence of the IEC, it cannot distinguish between the different contributions, i.e., it cannot yield (11) in the form $F_{\mathrm{IEC}}(T)=F_{\text {spacer }}(T)+F_{\text {magn. }}(T)$.

Figure 1 shows FMR experimental evidence (evaluated with LL) that the magnetic layer effect gives indeed a significant contribution. According to the theoretical works by Bruno [1] and Edwards et al. [2] the $T$-dependence should be more pronounced for a higher number of spacer layers while it should be independent of the spacer thickness within the spacer theory proposed in Ref. [9]. The observed stronger decrease of the 4-MLspacer (AFM coupled) system as compared to the 5-MLspacer (FM coupled) system suggests that there are other than just spacer mechanisms at work. Moreover one sees a large difference between the measured $J_{\text {inter-curve of }}$ the 4-ML-spacer film and the theoretical curve according to the spacer theory suggested in Ref. [1].

Ferromagnetic resonance is a powerful tool to investigate the IEC experimentally [12]. This technique probes the uniform spin wave mode $\omega(\mathbf{q}=0)$ of a magnetic 


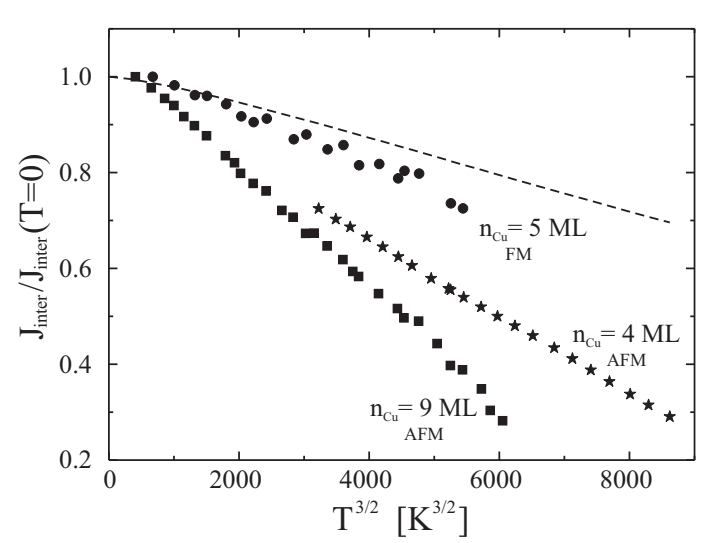

FIG. 1: Temperature dependence of $J_{\text {inter }}$ normalized to $J_{\text {inter }}(T=0)$ for the trilayer IEC system $\mathrm{Ni}_{7} / \mathrm{Cu}_{n} / \mathrm{Co}_{2} / \mathrm{Cu}(001)$ for three different spacer thicknesses $n_{\mathrm{Cu}}$ 14]. The data for $9 \mathrm{ML}$ (full squares) are taken from Ref. 5. The dashed line corresponds solely to a spacer effect contribution for the $n_{\mathrm{Cu}}=4 \mathrm{ML}$ sample.

sample. An external field $\mathbf{B}_{0}$ is tuned for a given probe frequency $\nu_{\mathrm{hf}}=\omega(\mathbf{q}=0) / 2 \pi$ until resonance occurs at $B_{\text {res }}\left(T, \theta_{B_{0}}\right)$, with $\theta_{B_{0}}$ being the angle between the magnetic field and the normal to the film plane. This resonance field $B_{\text {res }}\left(T, \theta_{B_{0}}\right)$ at which uniform $(\mathbf{q}=0)$-spin wave modes with the energy $E^{\mathrm{SW}}=h \nu_{\mathrm{hf}}$ of the probing microwaves are excited in the magnetic system is the crucial quantity to connect experiment and theory. With the method we propose it is possible to compute the spin wave energy $E^{\mathrm{SW}}\left(\mathbf{q}=0, \mathbf{B}_{0}, T\right)$ and read off the resonance field given the (experimental) parameter $\nu_{\mathrm{hf}}$, which was set to $9 \mathrm{GHz}$ in our calculations [13]. For further details about the experimental procedure we refer to Ref. 12] and give here a short outline of the experimental procedure. After the preparation of the $\mathrm{Cu}_{n} / \mathrm{Co}_{2} / \mathrm{Cu}(001)$ single film already capped with $n \mathrm{Cu}$ spacer layers, the angular dependence of the resonance field $B_{\mathrm{res}}^{\mathrm{Co}}\left(T, \theta_{B_{0}}\right)$ was determined at two temperatures $(55 \mathrm{~K}$ and $294 \mathrm{~K})$. Then the temperature dependence of the resonance field $B_{\text {res }}^{\mathrm{Co}}\left(T, \theta_{B_{0}}\right)$ with $T \in[55 \mathrm{~K}, \approx 300 \mathrm{~K}]$ at $\theta_{B_{0}}=90^{\circ}$ was measured. The same was carried out after the deposition of the top nickel layer for the coupled system $\mathrm{Ni}_{7} / \mathrm{Cu}_{9} / \mathrm{Co}_{2} / \mathrm{Cu}(001)$ and for comparison also for the single film system $\mathrm{Ni}_{7} / \mathrm{Cu}(001)$.

The Hamiltonian of the effective two-layer system we use to describe IEC reads

$$
\begin{aligned}
H= & -\sum_{<i j>\alpha} J_{\alpha} \mathbf{S}_{i \alpha} \mathbf{S}_{j \alpha}-\sum_{i \alpha} g_{\alpha} \mu_{\mathrm{B}} \mathbf{B}_{0} \mathbf{S}_{i \alpha}- \\
& -\sum_{<i j>\alpha \beta}^{\alpha \neq \beta} J_{I} \mathbf{S}_{i \alpha} \mathbf{S}_{j \beta}-\sum_{i \alpha} K_{2 \alpha} S_{i \alpha z}^{2}+ \\
& +\sum_{i j \alpha} g_{0 \alpha}\left(\frac{1}{r_{i j}^{3}} \mathbf{S}_{i \alpha} \mathbf{S}_{j \alpha}-\frac{3}{r_{i j}^{5}}\left(\mathbf{S}_{i \alpha} \mathbf{r}_{i j}\right)\left(\mathbf{S}_{j \alpha} \mathbf{r}_{i j}\right)\right)
\end{aligned}
$$

The first term describes nearest-neighbor exchange cou-

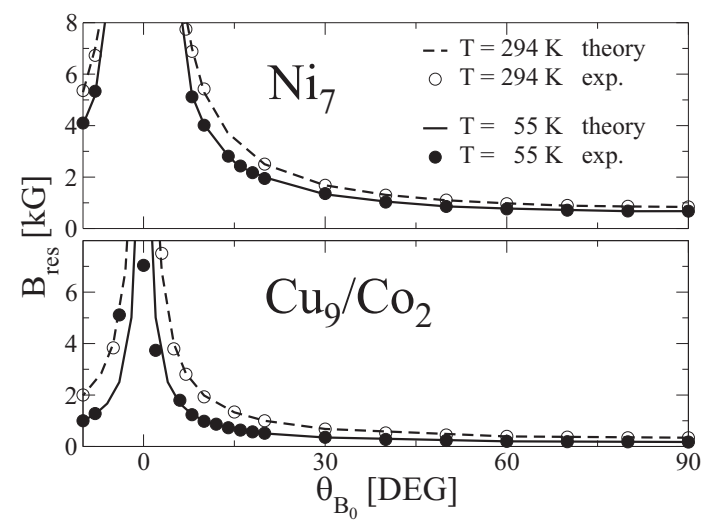

FIG. 2: Resonance field for $\mathrm{Ni}_{7} / \mathrm{Cu}(001)$ and $\mathrm{Cu}_{9} / \mathrm{Co}_{2} / \mathrm{Cu}(001)$ as a function of the orientation of the external magnetic field (circles). The angle $\theta_{B_{0}}$ is measured with respect to the film normal. Lines are theoretical fits, $\mathrm{Ni}: S=1, K_{2}=3.0 \mu_{\mathrm{B}} \mathrm{kG}, g_{0}=4.5 \mu_{\mathrm{B}} \mathrm{kG}, J=30 \mathrm{meV}$, Co: $S=2.5, K_{2}=-20.25 \mu_{\mathrm{B}} \mathrm{kG}, g_{0}=1.95 \mu_{\mathrm{B}} \mathrm{kG}, J=4.1 \mathrm{meV}$.

pling $J_{\alpha}$ between spin moments $\mathbf{S}_{i \alpha}$ and $\mathbf{S}_{j \alpha}$ at sites $i$ and $j$ within the same layer $\alpha(=1,2)$. The film thickness is implicitly included in the parameters $J_{\alpha}$ which are chosen such that the magnetic moments of the two monolayers described in (2) equal that of the considered multilayer films at room temperature, respectively $\left(T_{\mathrm{C}}^{\mathrm{Ni}_{7}}=410 \mathrm{~K}, T_{\mathrm{C}}^{\mathrm{Co}_{2}}=400 \mathrm{~K}\right)$. The second term contains an external magnetic field $\mathbf{B}_{0}$ in arbitrary direction with the spectroscopic splitting factors $g_{\alpha}$ (taken as 2.2 for $\mathrm{Ni}$ and $\mathrm{Co}$ ) and the Bohr magneton $\mu_{\mathrm{B}}$. The spacer layer is not considered explicitly. The IEC mediated by the spacer is described by the parameter $J_{I}$ (third term). The fourth and fifth term represent second order lattice anisotropy and dipolar interaction, the latter leading to shape anisotropy. $K_{2 \alpha}$ and $g_{0 \alpha}$ are microscopic anisotropy parameters, $S_{i \alpha z}$ is the $z$-component of $\mathbf{S}_{i \alpha}$ and is perpendicular to the film plane, $r_{i j}$ is the distance between sites $i$ and $j$, and dipolar coupling across the spacer is neglected. The shape anisotropy favors in-plane orientation of the magnetization, the lattice anisotropy in-plane $\left(K_{2 \alpha}<0\right)$ or out-of-plane $\left(K_{2 \alpha}>0\right)$ orientation. Our Hamiltonian is similar to that used in Ref. [15] for the investigation of magnetic reorientation and anisotropy. We also point out that the local-moment aspect of inherently itinerant magnets like $\mathrm{Ni}$ and Co has been demonstrated before: we mention here Heisenberglike spin wave dispersions of an itinerant band model [16], a high degree of the $d$-band moment localization $(>90 \%$ for $0<T<T_{\mathrm{C}}$ ) in an LDA + many-body approach to bulk Ni [17], and the successful employment of the Heisenberg model with ab-initio exchange parameters for the calculation of the Curie temperature of $\mathrm{Ni}[18]$.

We summarize shortly how we solved (2) for the singlemagnon Green function $(\mathrm{GF})\left\langle\left\langle S_{i \alpha}^{+} ; S_{j \beta}^{-}\right\rangle\right\rangle$and refer to Refs. 13, 19, 20] for details. In order to treat the 


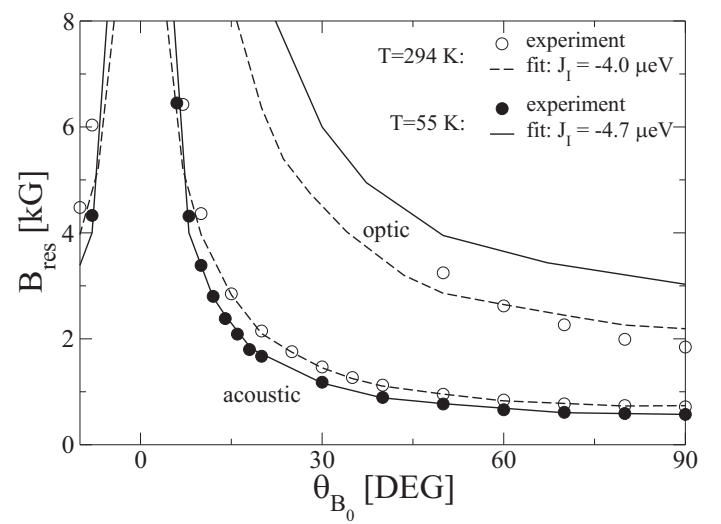

FIG. 3: Resonance field for the coupled system $\mathrm{Ni}_{7} / \mathrm{Cu}_{9} / \mathrm{Co}_{2} / \mathrm{Cu}(001)$. All parameters for the $\mathrm{Ni}$ and Co subsystems are the same as in Fig. 2 .

anisotropy terms properly we rotate the coordinate system self-consistently such that the new z-axis is parallel to the direction of the magnetization. In the subsequent equation of motion approach it is important to include all four combinations of the GF $\left\langle\left\langle S_{i \alpha}^{+,-} ; S_{j \beta}^{+,-}\right\rangle\right\rangle$as was first suggested in Ref. [20]. This ensures correct softening properties of the uniform spin wave mode, namely its vanishing at the reorientation field for $K_{2}>0$ and up to a critical field strength for $K_{2}<0$, respectively. Higher order GFs stemming from the Heisenberg exchange and from the dipolar interaction are decoupled using the Tyablikov (RPA) method. In the case of the dipoledipole interaction only the uniform $(\mathbf{q} \rightarrow 0)$ contribution is considered as the nonuniform terms are negligible compared to contributions from the much larger Heisenberg exchange. For the local $K_{2}$-terms an RPA decoupling fails. It was shown in Ref. [19] that the Anderson-Callen decoupling yields quantitative agreement with QMC results for the field induced magnetic reorientation transition. This is also the case for our improved theory which combines the proposal of Ref. [19] with the multiple GF approach by Pini et al. [20]. Different from the theoretical treatment based on the Landau-Lifshitz equations, now with the quantum Heisenberg model a self-consistent evaluation of the magnetization and the spin wave excitation spectrum $E^{\mathrm{SW}}\left(\mathbf{q}, \mathbf{B}_{0}, T\right)$ is carried out.

In our theory the anisotropies $K_{2 \alpha}$ and $g_{0 \alpha}$ influence the system solely via the effective anisotropy field given by the temperature-dependent term

$$
\begin{gathered}
\tilde{K}_{2 \alpha}(T)=\left\langle S_{z \alpha}\right\rangle(T)\left(2 K_{2 \alpha} C_{\alpha}(T)-D g_{0 \alpha}\right) \\
C_{\alpha}(T)=1-\frac{\left(S(S+1)-\left\langle S_{z \alpha}^{2}\right\rangle(T)\right)}{2 S 2} .
\end{gathered}
$$

Here the $T$-independent quantity $D$ is some number depending on the lattice geometry. Note that our effective anisotropy field $\tilde{K}_{2 \alpha}$ corresponds to the quantity $M_{\text {eff }}$ commonly used within a Landau-Lifshitz description of FMR experiments [12]. Furthermore we exploit

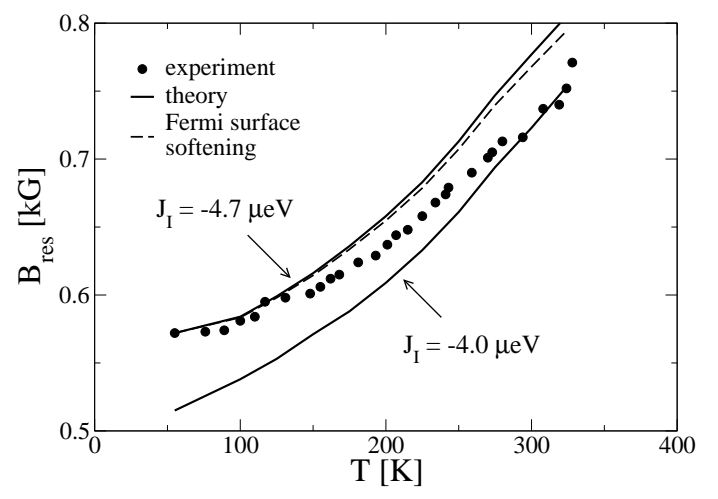

FIG. 4: Resonance field at $\theta_{B_{0}}=90^{\circ}$ as a function of temperature for $\mathrm{Ni}_{7} / \mathrm{Cu}_{9} / \mathrm{Co}_{2} / \mathrm{Cu}(001)$. Same parameters for $\mathrm{Ni}$ and Co as in Figs. 2 and 3 . Dashed line: the $T$-dependence due to the softening of the $\mathrm{Cu}$ Fermi surface [1] was included.

$\left\langle S_{z \alpha}^{2}\right\rangle(T)=S(S+1)-\left\langle S_{z \alpha}\right\rangle(T)\left[1+2 \varphi_{\alpha}(T)\right]$ with the average magnon occupation number $\varphi_{\alpha}$ [13]. The $T$ dependence of $\tilde{K}_{2 \alpha}$ goes beyond a mere proportionality to $\left\langle S_{z \alpha}\right\rangle(T)$ due to the expectation value $\left\langle S_{z \alpha}^{2}\right\rangle(T)$.

Figure 2 shows the comparison between the $B_{\text {res }}\left(\theta_{B_{0}}\right)$ curves from theory and experimental data for a $\mathrm{Cu}_{9} / \mathrm{Co}_{2} / \mathrm{Cu}(001)$ and $\mathrm{a}_{7} / \mathrm{Cu}(001)$ film system at two different temperatures. In both cases the effective anisotropy favors the magnetization to lie within the film plane. There is quite good agreement at both temperatures over the whole range of angles $\theta_{B_{0}}$ for both films.

It is important to note that for a fixed temperature the $B_{\text {res }}\left(\theta_{B_{0}}\right)$ data alone do not allow one to determine the anisotropy parameters $K_{2 \alpha}$ and $g_{0 \alpha}$ independently of each other [see Eq. (3)]. But taking into account both the $B_{\text {res }}\left(\theta_{B_{0}}\right)$ data and $B_{\text {res }}(T)$ at fixed $\theta_{B_{0}}=90^{\circ}$ the parameters can be separated because of the temperature dependent factor $C_{\alpha}(T)$ in Eq. (3). Indeed it is still possible to accurately fit the experimental results with one set of ( $T$-independent) parameters $\left(K_{2 \alpha}, g_{0 \alpha}\right)$ for $\mathrm{Ni}$ and Co, respectively, over the whole temperature range. Furthermore in both cases the values of $g_{0 \alpha} S$ lie slightly above the result of an explicit evaluation of this quantity assuming point-like dipoles on the lattice sites for the given geometry [12] $\left(g_{0}^{*} S=3.81 \mu_{\mathrm{B}} \mathrm{kG}\right)$. The conclusion we draw is that the $T$-dependence of the effective magnetic anisotropy is solely due to spin wave excitations which manifest themselves in the $T$-dependence of (3) rather than due to $T$-dependent $K_{2 \alpha}, g_{0 \alpha}$ which would effectively describe nonmagnonic sources as thermal expansion or phononic interactions. This means that we only have one fit parameter, $J_{I}$, for the IEC system.

Figure 3 shows the resonance fields for such a film system. The two branches at a given $T$ correspond to the acoustic (lower field branch) and optical (high field branch) excitation modes, respectively. The optical mode lying above the acoustic indicates antiferromagnetic coupling between $\mathrm{Ni}$ and $\mathrm{Co}$. The missing experimental data 


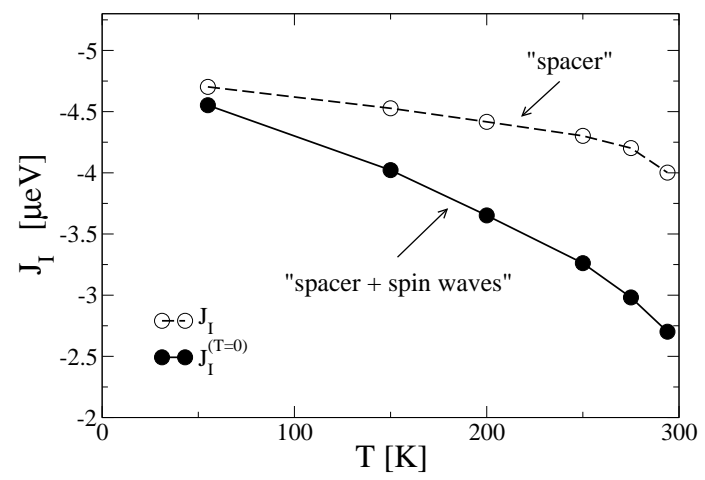

FIG. 5: Temperature dependence of interlayer exchange coupling in $\mathrm{Ni}_{7} / \mathrm{Cu}_{9} / \mathrm{Co}_{2} / \mathrm{Cu}(001)$. Symbols stem from fits of the experimental resonance field at $\theta=90^{\circ}$ at the given temperatures. Open circles: $T$-dependent calculation. Filled circles: calculation at $T=0$. Lines are guides to the eye.

for the optical mode at $T=55 \mathrm{~K}$ are due to a low intensity (oscillator strength) of the resonance signal [12]. All parameters except for $J_{I}$ stem from the fits of the single (uncoupled) $\mathrm{Ni}$ and Co films in Fig. 2 ,

In addition we carried out fits at a fixed angle $\theta=90^{\circ}$ for a variety of temperatures, the results of which are

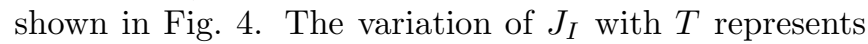
the spacer contributions. From comparison with the results obtained by additionally taking Fermi surface softening into account we conclude that sources other than that dominate the spacer contribution, presumably the $T$-dependence of the reflection coefficients.

In order to separate quantitatively the spacer and the magnetic $T$-dependence of the IEC we fitted in a sec-

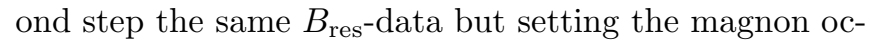
cupation number in our model artificially to zero, i.e., we "switched off" the magnons. The whole temperature dependence of the IEC including magnon excitations is then accounted for by the fit parameter $J_{I}^{(0)}$. From the comparison between both resulting curves of $J_{I}(T)$ and $J_{I}^{(0)}(T)$ in Fig. 5 lone can read off the weight of each contribution to the $T$-dependence of the IEC. One concludes that the magnonic part $(\approx 75 \%)$ exceeds the spacer part $(\approx 25 \%)$ significantly, however the latter is not negligible.

As for the variation of both contributions with the number of magnetic and non-magnetic layers one can state the following general trend: the fewer spacer layers and the thinner the magnetic part (i.e. the smaller $T_{C}$ ), the more important spin waves become for IEC. Of course, for bulk-like systems of Fe or Co with $T_{C}^{\text {bulk }}>$ $1000 \mathrm{~K}$ the magnon part will be (possibly much) less significant at room temperature or less. However, for $T_{C}$ below $\approx 700 \mathrm{~K}$, as for thin nano-film systems or Ni films or manganite multilayers [21], the spin wave contribution to the IEC temperature dependence becomes dominant.

In conclusion we proposed a novel approach that combines a quantum theoretical treatment of the magnetic properties of multilayer film systems with FMR experi- ments on prototype $\mathrm{Ni}_{7} / \mathrm{Cu}_{n} / \mathrm{Co}_{2} / \mathrm{Cu}(001)$ interlayer exchange coupled films. Employing a microscopic theory based on an extended Heisenberg Hamiltonian we studied the temperature dependence of the IEC by fitting experimental data on the FMR resonance field in two steps, first with $T$-dependent calculations and then with $T=0$; thus, "switching off" magnon excitations in the system. The result is that magnon excitations are most important $(\approx 75 \%)$ for the temperature dependence of the interlayer exchange coupling. Band structure effects (e.g. smearing of Fermi surface) are present but of minor importance. As a result we note that a theoretical treatment of IEC at $T=0$ is of limited use when interpreting the IEC of real multilayers at temperatures relevant for technological applications.

Financial support by the DFG (Sfb 290) is gratefully acknowledged.

* Corresponding author, kienert@physik.hu-berlin.de

$\dagger$ new address: Fachbereich Physik, Experimentalphysik, Universität Duisburg-Essen, Lotharstr. 1, 47048 Duisburg, Germany

[1] P. Bruno, Phys. Rev. B. 52, 411 (1995).

[2] D.M. Edwards et al., Phys. Rev. Lett. 67, 493 (1991).

[3] M.D. Stiles in Ultrathin magnetic Structures III, edited by J.A.C. Bland and B. Heinrich (Springer Berlin, 2005).

[4] J. Unguris et al., Phys. Rev. Lett. 79, 2734 (1997).

[5] J. Lindner, C. Rüdt, E. Kosubek, P. Poulopoulos, K. Baberschke, P. Blomquist, R. Wäppling, and D. L. Mills, Phys. Rev. Lett. 88, 167206 (2002).

[6] Z. Zhang et al., Phys. Rev. Lett. 73, 336 (1994); Phys. Rev. B 50, 6094 (1994).

[7] N. Persat and A. Dinia, Phys. Rev. B 56, 2676 (1997).

[8] V. Drchal, J. Kudrnovsky, P. Bruno, I. Turek, P.H. Dederichs, and P. Weinberger, Phys. Rev. B. 60, 9588 (1999).

[9] J. d'Albuquerque e Castro, J. Mathon, M. Villeret, and A. Umerski, Phys. Rev. B 53, R13306 (1996).

[10] N.S. Almeida, D.L. Mills, and M. Teitelman, Phys. Rev. Lett. 75, 733 (1994).

[11] S. Schwieger and W. Nolting, Phys. Rev. B. 69, 224413 (2004).

[12] J. Lindner and K. Baberschke, J. Phys.: Condens. Matter 15 R193 (2003); ibid. S465 (2003).

[13] S. Schwieger, J. Kienert, and W. Nolting, Phys. Rev. B 71, 174441 (2005).

[14] K. Lenz, Ph.D. thesis, Freie Universität Berlin (dissertation.de, 2005), ISBN 3-86624-013-9.

[15] Huai-Yu Wang et al., Phys. Rev. B 70, 134424 (2004).

[16] J. Bünemann and F. Gebhard, J. Phys.: Condens. Matter 13, 9985 (2001).

[17] W. Nolting et al., Phys. Rev. B. 40, 5015 (1989).

[18] M. Pajda et al., Phys. Rev. B. 64, 174402 (2001).

[19] S. Schwieger, J. Kienert, and W. Nolting, Phys. Rev. B 71, 024428 (2005).

[20] M.G. Pini et al., Phys. Rev. B 72, 014454 (2005).

[21] T.G. Perring et al., Phys. Rev. Lett. 87, 217201 (2001); T. Ohsawa et al., Phys. Rev. B 71, 212407 (2005). 